\title{
Electro-thermal and optical characterization of an uncooled suspended bolometer based on an epitaxial $\mathrm{La}_{0.7} \mathrm{Sr}_{0.3} \mathrm{MnO}_{3}$ film grown on $\mathrm{CaTiO}_{3} / \mathrm{Si}$
}

\author{
V.M. Nascimento, ${ }^{1}$ L. Méchin, ${ }^{1}$ S. Liu, ${ }^{1}$ A. Aryan, ${ }^{1}$ C. Adamo, ${ }^{2,3}$ D.G. Schlom, ${ }^{3,4,5}$, and \\ B. Guillet ${ }^{1}$
}

${ }^{1}$ Normandie Univ. UNICAEN, ENSICAEN, CNRS, GREYC, 14000 Caen, France,

${ }^{2}$ Department of Materials Science and Engineering, Cornell University, Ithaca, New York 14853-1501, USA

${ }^{3}$ Department of Applied Physics, Stanford University, Stanford, California 94305, USA

${ }^{4}$ Kavli Institute at Cornell for Nanoscale Science, Cornell University, Ithaca, New York 14853-1501, USA

${ }^{5}$ Leibniz-Institut für Kristallzüchtung, Max-Born-Str. 2, 12489 Berlin, Germany

\section{Abstract}

The electro-thermal and optical properties of a bolometer based on an $\mathrm{La}_{0.7} \mathrm{Sr}_{0.3} \mathrm{MnO}_{3}$ (LSMO) thin film with a detection area of $100 \times 100 \mu \mathrm{m}^{2}$ are presented. The LSMO thin film was epitaxially grown on $\mathrm{CaTiO}_{3} / \mathrm{Si}$ and patterned using a two-step etching process of ion-beam etching in argon and of reactive-ion etching in $\mathrm{SF}_{6}$, in order to etch $\mathrm{LSMO} / \mathrm{CaTiO}_{3}$ and $\mathrm{Si}$, respectively. The voltage-current (V-I) characteristics of the bolometer were measured in vacuum from 240 to $415 \mathrm{~K}$. From the V-I characteristics and a thermal model of the bolometer, the electrical responsivity was determined and compared to the optical responsivity measured with a laser diode at $635 \mathrm{~nm}$. The noise equivalent power (NEP) as a function of frequency was measured by dividing the spectral noise power density by the optical responsivity. At $300 \mathrm{~K}$ and a bias current of $80 \mu \mathrm{A}$, the NEP was $2.3 \times 10^{-11} \mathrm{~W} \cdot \mathrm{Hz}^{-1 / 2}$ in the $20-200 \mathrm{~Hz}$ modulation frequency range and the response time was $1.3 \mathrm{~ms}$. The obtained NEP value without any absorbing layer or antennas, combined with the low value of the response time, are a very promising step towards the use of such LSMO-based bolometers for IR or THz detection. 


\section{Introduction}

Infrared and $\mathrm{THz}$ detectors may have important applications in medical or security imaging, environmental sensing, material detection, non-destructive testing, and astronomy $[1,2,3,4,5]$. Several physical mechanisms can be used to detect radiation, resulting in detectors in two main categories: thermal and electronic (or photonic) detectors. The latter rely on the interaction of the photons with electrons in the detector and the performance is very dependent on the wavelength to be detected, while in thermal detectors the absorbed energy increases the temperature, which can be measured by sensitive thermometers. The detection efficiency of such thermal detectors can be improved by increasing the absorption using absorbing layers or antennas. The change in temperature can be converted to a change of volume in golay cells, to a change in electric potential in pyroelectric detectors, or to a change of electrical resistance in semiconducting, superconducting, or metallic bolometers.

The main figures of merit for radiation detectors are optical responsivity $\left(\mathfrak{R}_{\mathrm{V}}\right)$ and noise equivalent power (NEP) [6,7]. The optical responsivity measures the variation of the electrical voltage or current output (if current or voltage biased, respectively), per optical power input and is expressed in $\mathrm{V} \cdot \mathrm{W}^{-1}$ or $\mathrm{A} \cdot \mathrm{W}^{-1}$. The NEP expresses the minimum detectable power per square root bandwidth of a given detector. It is the ratio between the noise power spectral density (in voltage $\mathrm{S}_{\mathrm{V}}$ in $\mathrm{V}^{2} \cdot \mathrm{Hz}^{-1}$ if current biased, or in current $\mathrm{S}_{\mathrm{I}}$ in $\mathrm{A}^{2} \cdot \mathrm{Hz}^{-1}$ if voltage biased) and the optical responsivity. It is desirable to have a NEP as low as possible, since a low NEP value corresponds to a lower noise floor and therefore a more sensitive detector. As shown in Table I, the lowest NEP values in the literature lie in the $10^{-9}-10^{-12} \mathrm{~W} \cdot \mathrm{Hz}^{-1 / 2}$ range for uncooled antennacoupled detectors such as Golay cells [8], pyroelectric detectors [9], Schottky-barrier diodes [10], GaN high electron mobility transistors [11], metallic, $\mathrm{VO}_{2}$, amorphous $\mathrm{Si}$ (a-Si), or SiGe microbolometers $[12,13,14,15]$.

The manganite material of composition $\mathrm{La}_{0.7} \mathrm{Sr}_{0.3} \mathrm{MnO}_{3}$ (LSMO) is a promising candidate to fabricate uncooled bolometers thanks to the large change in electrical resistance versus temperature (a metal-to-insulator transition) that it exhibits close to room temperature and the low-noise level compared with other resistive materials such as semiconductors (a-Si, a-Si:H, a$\mathrm{Ge}$, poly $\mathrm{SiGe}$ ) and other oxide materials (semiconducting $\mathrm{YBa}_{2} \mathrm{Cu}_{3} \mathrm{O}_{7-\delta}, \mathrm{VO}_{\mathrm{x}}$, etc.) $[16,17,18,19]$. The objective of this paper is to investigate the electrothermal properties of a device made of 17 suspended lines patterned in LSMO thin films that cover a detection area of 
$100 \times 100 \mu \mathrm{m}^{2}$, and to show that a new technology of uncooled bolometers based on LSMO can be developed. After a short description of the bolometer fabrication, the experimental methods and principle of operation, the voltage-current (V-I) characteristics measured in the 240-415 K range are presented. The electrical responsivity, which was calculated from the derivative of the electrical resistance versus temperature characteristics, was compared to the optical responsivity measured under illumination with a laser diode at $635 \mathrm{~nm}$. The difference between the optical and electrical responsivity is the optical coupling efficiency of the detector system. Finally, NEP values are deduced from the measured spectral noise power density and responsivity versus frequency curves.

Table I: Reported NEP values of uncooled detectors in the literature.

\begin{tabular}{|l|c|c|c|}
\hline Type of detector & $\begin{array}{c}\text { Operating wavelength or } \\
\text { frequency range }\end{array}$ & NEP $\left(\mathrm{W} \cdot \mathrm{Hz}^{-1 / 2}\right)$ & Reference \\
\hline Golay Cell & $0.3-8000 \mu \mathrm{m}$ & $1.4 \times 10^{-10}$ & {$[8]$} \\
\hline Pyroelectric detectors & $2.52 \mathrm{THz}$ & $1.5 \times 10^{-9}$ & {$[9]$} \\
\hline Schottky barriers & $100 \mathrm{GHz}-1 \mathrm{THz}$ & $7-100 \times 10^{-12}$ & {$[10]$} \\
\hline $\begin{array}{l}\text { GaN high electron mobility } \\
\text { transistors }\end{array}$ & $0.14 \mathrm{THz}$ & $0.58 \times 10^{-12}$ & {$[11]$} \\
\hline Metallic microbolometers & $1 \mathrm{THz}$ & $4.6 \times 10^{-10}$ & {$[12]$} \\
\hline VO microbolometers & $8-12 \mu \mathrm{m}$ & $1.54 \times 10^{-12}$ & {$[13]$} \\
\hline a-Si microbolometers & $10-12 \mu \mathrm{m}$ & $2.6 \times 10^{-12}$ & {$[14]$} \\
\hline $\begin{array}{l}\mathrm{Si}_{\mathrm{x}} \mathrm{Ge} \text { : } \mathrm{H}-\text { based } \\
\text { microbolometers }\end{array}$ & $0.934 \mathrm{THz}$ & $2 \times 10^{-10}$ & {$[15]$} \\
\hline
\end{tabular}

\section{Methods}

The $50 \mathrm{~nm}$ thick LSMO films were deposited on $20 \mathrm{~nm}$ thick $\mathrm{CaTiO}_{3}$ (CTO) buffers layers grown on silicon (001) substrates by reactive molecular-beam epitaxy as described in Adamo et al. [20]. The use of a buffer layer is necessary in order to prevent interdiffusion between Si and LSMO and specific procedures must be used in order to avoid the formation of amorphous $\mathrm{SiO}_{2}$ at the Si surface. In addition, the thickness of the LSMO layer is limited because of the large difference between its thermal expansion coefficient and that of Si. Further, the buffer layer 
material separating LSMO from Si and preventing them from reacting with one another should show a good lattice match with both. A variety of materials have been used to act as a buffer layer for the epitaxial growth of oxides on silicon as reviewed by Reiner et al. [21]. Our LSMO/CTO heterostructures were epitaxially grown on $\mathrm{Si}$ (001). They showed a (001) orientation, and slightly improved structural and electrical properties compared to high quality LSMO/STO layers grown on $\mathrm{Si}[22,23]$.

Only two photolithographic steps are needed in the fabrication process. The first defines the electrode pads using a potassium iodide solution and the second step defines the bridge geometry of the bolometer using argon ion beam milling down to the silicon substrate. The detailed fabrication process has been published previously [24,25]. Figure 1(a) shows an optical micrograph of the device studied; the 4 gold contacts for voltage and current pads can be seen. The detection area of the bolometer is made of 17 suspended LSMO/CTO lines, each of width 4 $\mu \mathrm{m}$ separated by about $2 \mu \mathrm{m}$, which covers a detection area of $100 \times 100 \mu \mathrm{m}^{2}$, as shown in Fig. 1(b). The lines were suspended by using the isotropic nature of reactive-ion etching (RIE) of the silicon in $\mathrm{SF}_{6}$ gas from the front side.

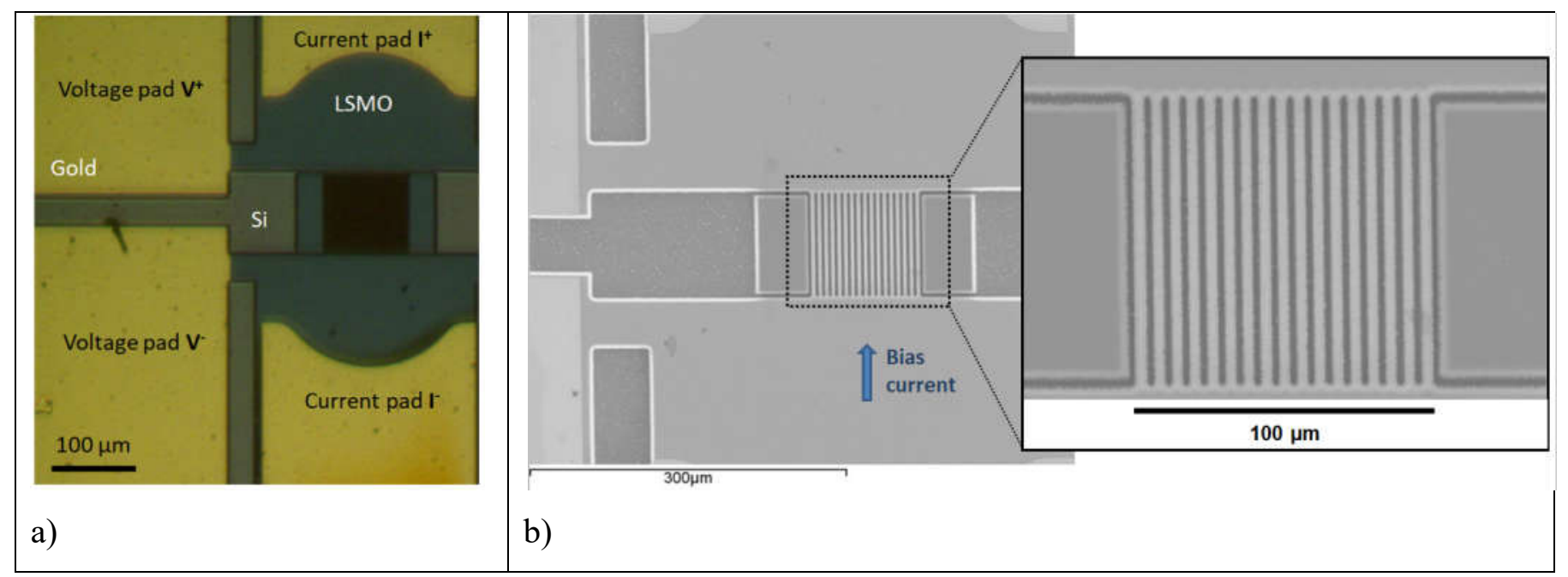

Figure 1: (a) Optical micrograph of the tested device showing the current and voltage pads as well as the $100 \times 100 \mu \mathrm{m}^{2}$ detection area; (b) Scanning electron microscope photography of the $100 \times 100 \mu \mathrm{m}^{2}$ detection area patterned in the LSMO/CTO/Si films. It consists of 17 suspended lines each of width $4 \mu \mathrm{m}$ separated by about $2 \mu \mathrm{m}$. The bias current I flows in parallel in the 17 lines. 
The experimental setup is based on a sample holder placed in a vacuum chamber (at a pressure below $10^{-3}$ mbar) and equipped with a $\mathrm{NaCl}$ window. Liquid nitrogen and a temperature controller using a platinum sensor and a simple proportional-integral (PI) control loop were used to set the sample temperature in the $230 \mathrm{~K}-415 \mathrm{~K}$ range. The V-I characteristics were measured with a semiconductor parameter analyzer (Agilent 4156). The optical measurements were performed using a power-modulated laser diode, by varying the current at the modulation frequency $\mathrm{f}$ (wavelength of $635 \mathrm{~nm}$ and nominal power of $5 \mathrm{~mW}$ ). The radiation beam at the output of the laser diode passed through a beam splitter cube, which separated the beam into two paths. One beam is incident on the sample and the other one on a photodiode used as a reference [26]. The laser beam that arrives on the sample was focused and highly attenuated. The power received by the LSMO bolometer was estimated in real time by knowing the transmission coefficients of the different optical elements and the output signal of the photodiode. It was about $23 \mu \mathrm{W}$ in the experiments presented here.

A homemade quasi-ideal DC current source was used for bias current, which has high output impedance and negligible input current noise [27]. A homemade voltage amplifier circuit based on a 3 Op-Amp AD743 instrumentation amplifier was used, with a noise floor of $4 \times 10^{-16} \mathrm{~V}^{2} \cdot \mathrm{Hz}^{-}$ ${ }^{1}$, in the $1 \mathrm{~Hz}-100 \mathrm{kHz}$ bandwidth, negligible input current noise (lower than $10^{-15} \mathrm{~V}^{2} \cdot \mathrm{Hz}^{-1}$ ), and gain of about 1000. Measurements of the optical responsivity versus frequency and noise spectral density, in the range of $1 \mathrm{~Hz}-2 \mathrm{kHz}$, were made using a spectrum analyzer (HP3562A). Finally, the optical responsivity could be estimated by dividing the output voltage by amplifier gain and the optical power received by the device.

The principle of operation of a bolometer and the figure of merits of bolometers are described in reference books [28,29,30]. If the bolometer is current biased, the optical responsivity at the laser modulation frequency $\mathrm{f}$ can be written as:

$$
\mathfrak{R}_{\mathrm{V}}(\mathrm{T}, \mathrm{I}, \mathrm{f})=\frac{\Delta \mathrm{V}(\mathrm{f})}{\Delta \mathrm{P}(\mathrm{f})}=\frac{\eta \mathrm{I}}{\mathrm{G}_{\mathrm{eff}}(\mathrm{T}, \mathrm{I}) \sqrt{1+\left(2 \pi \mathrm{f} \tau_{\mathrm{eff}}\right)^{2}}} \frac{\mathrm{dR}}{\mathrm{dT}}(\mathrm{T}, \mathrm{I})
$$

where $\eta$ is the absorption coefficient (dimensionless), $I$ is the bias current (in A), R is the electrical resistance (in $\Omega$ ), $\mathrm{G}_{\text {eff }}$ is the effective thermal conductance of the device (in $\mathrm{W} \cdot \mathrm{K}^{-1}$ ), $\tau_{\text {eff }}=\mathrm{C} / \mathrm{G}_{\text {eff }}$ is the effective thermal time constant (in s), and $\mathrm{C}$ is the thermal capacitance (in $\mathrm{J} \cdot \mathrm{K}^{-1}$ ). The thermal conductance measures the ability of the bolometer to transfer the absorbed 
power given a temperature gradient. The effective thermal conductance $\mathrm{G}_{\text {eff }}$ is defined, by taking into account the geometrical thermal conductance $\mathrm{G}$ and the self-heating effect, as:

$$
\mathrm{G}_{\text {eff }}(\mathrm{T}, \mathrm{I})=\mathrm{G}(\mathrm{T}, \mathrm{I})-\mathrm{I}^{2} \frac{\mathrm{dR}}{\mathrm{dT}}(\mathrm{T}, \mathrm{I})
$$

In order to maximise the optical responsivity, we need to choose materials showing high $\frac{\mathrm{dR}}{\mathrm{dT}}$, i.e., sensitive thermometers, to design geometries with low thermal conductance, i.e., suspended structures, and to choose appropriate operating conditions in temperature and bias current. In addition, to keep the thermal time constant low, a small thermal capacitance is required. Suspended thin films are then a good solution for achieving both high optical responsivity and low thermal response time (i.e., high cut-off frequency), by reducing simultaneously both $\mathrm{G}_{\text {eff }}$ and C. Many of the parameters depend on both the temperature and the bias current, which makes the analytical resolution of Eq. (1) intractable. In addition, the electrical resistivity in LSMO strongly depends on temperature with a nonlinear temperature dependence $[31,16]$, which should result in nonlinear operating conditions of the LSMO bolometers. For suspended lines, the thermal conductance is strongly reduced, which is expected to result in strong self-heating due to Joule heating when biasing the devices. Self-heating induces a local increase of the temperature, and will change the operating point and affect directly the figures of merit of the detector.

\section{Results and discussion}

The V-I characteristics of the 17 suspended lines were measured at different temperatures between $230 \mathrm{~K}$ and $415 \mathrm{~K}$ for bias currents in the $10 \mu \mathrm{A}-300 \mu \mathrm{A}$ range. Figure 2 presents the V-I curves, showing non linearities induced by the change of the average temperature of the suspended lines, thus affecting its electrical resistance. The temperature profile of the suspended lines has a parabolic shaped distribution along the bridge length $[32,33]$. When the bias current is high enough, regions in the centre of the suspended lines may reach the metal-to-insulating transition temperature. From the measured V-I characteristics and the electrical resistance versus temperature characteristics measured at reduced bias current $(10 \mu \mathrm{A})$, it is possible to know the temperature of the tested device as a function of the electrical power in the device, and therefore to estimate the thermal conductance $\mathrm{G}$, to be $(5.0 \pm 2.5) \times 10^{-6} \mathrm{~W} \cdot \mathrm{K}^{-1}$ at $300 \mathrm{~K}$. With the samples 
being in vacuum, radiative and convective leakage is negligible and the thermal conductance can be accurately considered to describe only the heat conduction from the film to the environment.

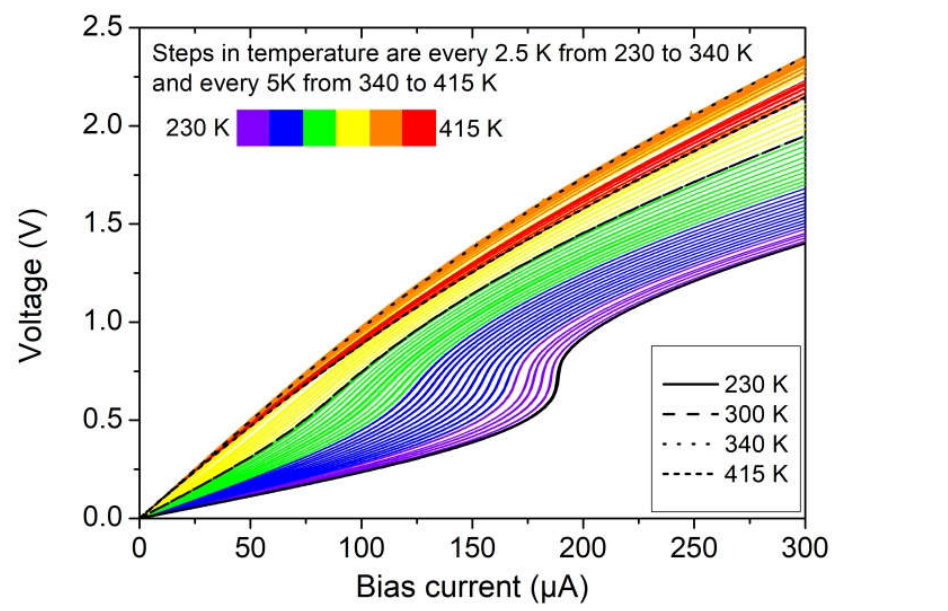

Figure 2: Voltage versus bias current characteristics measured in the 17 suspended lines at different temperatures. Steps in temperature are every $2.5 \mathrm{~K}$ from $230 \mathrm{~K}$ to $340 \mathrm{~K}$ and every $5 \mathrm{~K}$ from $340 \mathrm{~K}$ to $415 \mathrm{~K}$.

The electrical resistance characteristics versus temperature, $R(T, I)$, of the 17 suspended LSMO lines are reported in Fig. 3(a) for different bias currents in the $10-300 \mu \mathrm{A}$ range. At a bias current of $10 \mu \mathrm{A}$, a large change of the electrical resistance is observed at the metal-toinsulator transition temperature, with $\mathrm{T}_{\mathrm{P}}$, defined as the temperature of the maximum of resistance, close to $360 \mathrm{~K}$. This confirms that the fabrication process did not significantly degrade the electrical transport properties of the LSMO film. When the bias current is increased, the metal-to-insulator transition, and therefore $T_{P}$ and the large change of the electrical resistance, are shifted to the lower temperatures. The latter effect can be observed more clearly in Fig. 3(b), which plots the derivative of $R(T, I)$ versus $T, \frac{d R}{d T}(T, I)$ from the $R(T, I)$ curves of Fig. 3(a). It should be noted that at high current the device is not in thermal equilibrium and that the measured temperature is not the true temperature of the material in the suspended area but only gives the holder temperature. The maximum value of $\frac{d R}{d T}(T, I)$ slightly increases when the bias current increases and occurs at lower temperature. At $300 \mathrm{~K}$ and $80 \mu \mathrm{A}$, the value of $\frac{\mathrm{dR}}{\mathrm{dT}}(\mathrm{T}, \mathrm{I})$ is equal to $123 \Omega \cdot \mathrm{K}^{-1}$ corresponding to a temperature coefficient of the resistance $\left(\mathrm{TCR}=\frac{1}{\mathrm{R}} \times \frac{\mathrm{dR}}{\mathrm{dT}}\right)$ of $1.8 \times 10^{-2} \mathrm{~K}^{-1}$. This is comparable to the values for LSMO found in literature [34]. Figure 3(c) 
shows the electrical responsivity of the bolometer versus temperature, named $\mathfrak{R}_{\mathrm{V} \text {-elec }}(\mathrm{T}, \mathrm{I})$, calculated using Eq. (1) and the $\frac{d R}{d T}(T, I)$ characteristics plotted in Fig. 3(b), and assuming $\eta=1$. It should be noted that the bolometer can be operated over a large range of temperature, by an appropriate choice of bias current. Higher electrical responsivity values are obtained at low temperature, but at higher bias current. It is $85688 \mathrm{~V} \cdot \mathrm{W}^{-1}$ at $240 \mathrm{~K}$ and $175 \mu \mathrm{A}$ and $2327 \mathrm{~V} \cdot \mathrm{W}^{-1}$ at $300 \mathrm{~K}$ and $80 \mu \mathrm{A}$. Joule heating can be used to reach the maximum of $\frac{\mathrm{dR}}{\mathrm{dT}}(\mathrm{T}, \mathrm{I})$ at any temperature but to the detriment of higher power consumption, and higher low frequency noise, as will be discussed below.

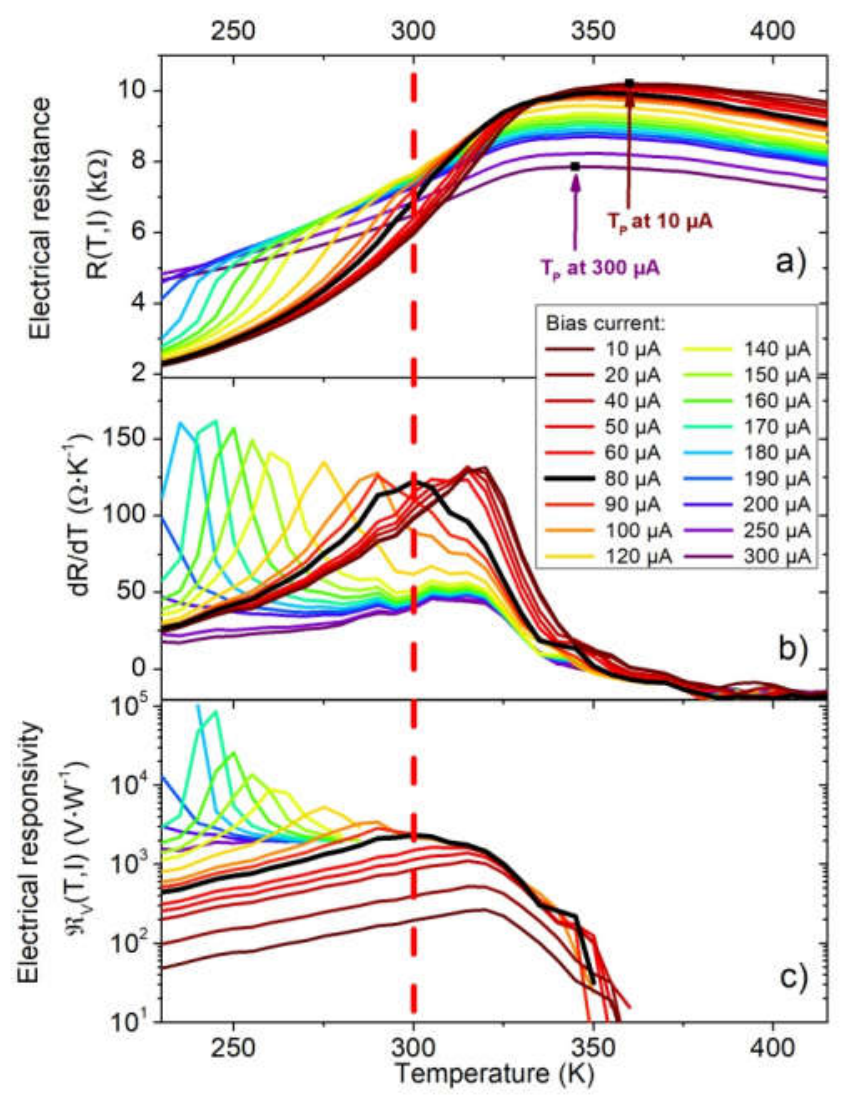

Figure 3: (a) Electrical resistance, $\mathrm{R}(\mathrm{T}, \mathrm{I})$, where the temperature of the maximum of resistance $\mathrm{T}_{\mathrm{P}}$ is indicated for a current of $10 \mu \mathrm{A}$ and $300 \mu \mathrm{A}$; (b) Derivative of the electrical resistance, $\frac{\mathrm{dR}}{\mathrm{dT}}(\mathrm{T}, \mathrm{I})$; (c) Electrical responsivity of the bolometer, $\mathfrak{R}_{\mathrm{V} \text {-elec }}(\mathrm{T}, \mathrm{I})$, measured as a function of the temperature and at different bias current in the $10 \mu \mathrm{A}-300 \mu \mathrm{A}$ range. The legend is valid for the 3 graphs. 


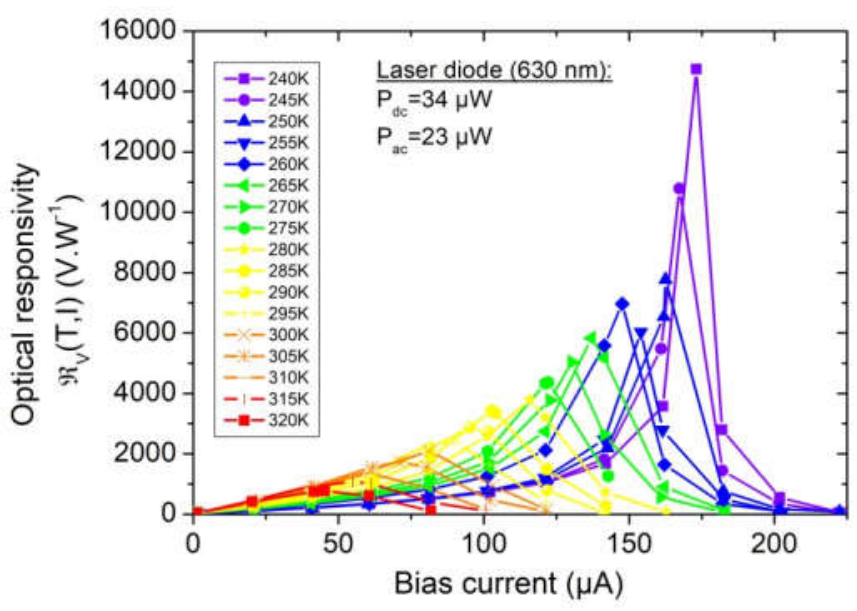

Figure 4: Optical responsivity $\mathfrak{R}_{\mathrm{V}}(\mathrm{T}, \mathrm{I})$ versus bias current at different temperatures in the bolometer bandwidth under illumination with incident radiation at $635 \mathrm{~nm}$.

Optical characterization has been performed at $635 \mathrm{~nm}$ using a low incident power (about 23 $\mu \mathrm{W})$ and at a modulation frequency of $8 \mathrm{~Hz}$, i.e., in the bandwidth of the bolometer, as will be shown in Fig. 6(a). Figure 4 shows the measured values versus bias current at fixed temperatures between $240 \mathrm{~K}$ and $320 \mathrm{~K}$. For each temperature, an optimal bias current can be defined, $\mathrm{I}_{\mathrm{opt}}$, at which the optical responsivity is maximum. For a thermal detector, the optical responsivity is equal to the electrical responsivity multiplied by the absorption coefficient of the detector $\eta$ at the considered radiation wavelength, which we measured to be $85 \%$ for LSMO at about $635 \mathrm{~nm}$ [35]. The precise optical power received by the device was difficult to evaluate with high precision, and in order to compare the optical and electrical responsivity, we normalized the measured values of the optical responsivity at $300 \mathrm{~K}$ by the electrical responsivity at $300 \mathrm{~K}$ multiplied by $\eta$. Both maximum values of the optical responsivity and electrical responsivity multiplied by $\eta$ have been plotted in Fig. 5 versus its optimal bias current. In the $60-120 \mu \mathrm{A}$ range (i.e., $310 \mathrm{~K}-275 \mathrm{~K}$ ), very good agreement is observed. Below and above this range, the discrepancies between the optical responsivity and the electrical responsivity can be accounted by different assumptions. At low bias current, the evaluation of the optical responsivity was difficult because it was very low. At high bias current, the self-heating effects are huge and the temperature is not uniform along the suspended lines, which induced inhomogeneities in the properties of LSMO that are not considered in the simple model of Eq. (1). Nonetheless, we can 
conclude that in the temperature range of interest around room temperature, Eq. (1) can be used to satisfactorily describe the bolometer responsivity.

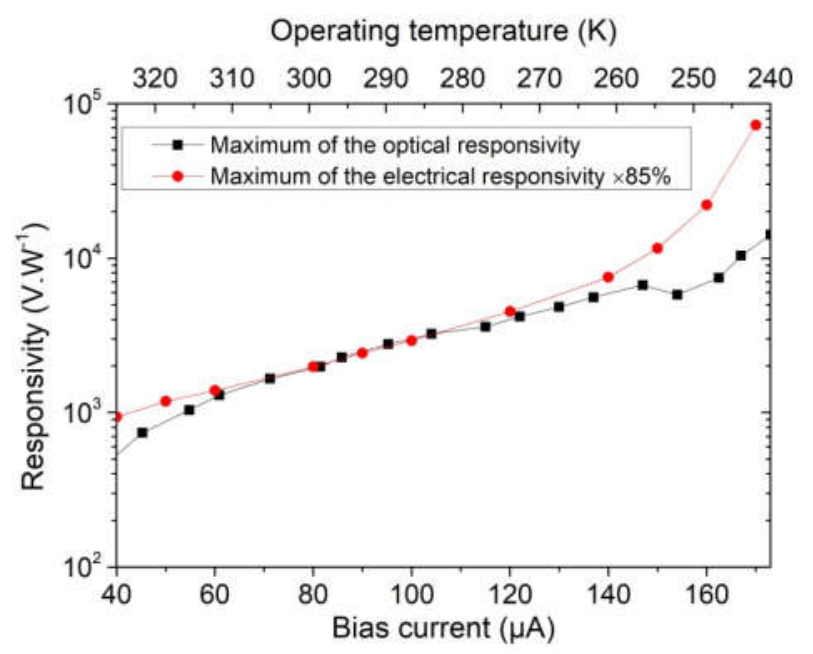

Figure 5: Maximum of the electrical and optical responsivity values versus bias current and temperature at which the maximum is measured. Electrical responsivity has been multiplied by $\eta=0.85$ and the optical responsivity has been superimposed upon the electrical responsivity value at $300 \mathrm{~K}$.

Figure 6(a) presents the optical responsivity of the device versus the modulation frequency measured at different temperatures and at the optimal current determined in Fig. 5. The bolometer behaves as a first-order thermal low-pass filter, having cut-off frequency at $-3 \mathrm{~dB}$ given by $\mathrm{f}_{-3 \mathrm{~dB}}=1 /\left(2 \pi \tau_{\text {eff }}\right)$, where $\tau_{\text {eff }}$ is the effective thermal time constant as defined in Eq. (1). The cut-off frequency is around $124 \mathrm{~Hz}$ at $300 \mathrm{~K}$ and $21 \mathrm{~Hz}$ at $240 \mathrm{~K}$, which corresponds to an effective thermal response time of $1.3 \mathrm{~ms}$ at $300 \mathrm{~K}$ and $7.6 \mathrm{~ms}$ at $240 \mathrm{~K}$. At higher frequencies one can observe an increase of the measured optical responsivity, which is independent of the bias current and which we attribute to photovoltaic effects in the silicon wafer. The input noise power spectral density in voltage was measured versus frequency at different temperatures and at the optimal current as shown in Fig. 6(b). Typically, the input noise power spectral density consists of two regions: a frequency and current dependent part at low frequency and a frequency and current independent part at high frequency, named white noise and equal to $4 \mathrm{k}_{\mathrm{B}} \mathrm{TR}$ (with $\mathrm{k}_{\mathrm{B}}$ the Boltzmann constant, $\mathrm{T}$ the temperature, and $\mathrm{R}$ the electrical resistance). As expected, the white noise decreases with decreasing temperature since both temperature and electrical resistance decrease. In contrast, the noise value at $1 \mathrm{~Hz}$ increases with decreasing temperature 
since the bias current is higher. Finally, in order to evaluate the bolometer performance, we estimated the NEP for different temperatures at the optimal bias current by dividing the curves of Fig. 6(b) by those of Fig. 6(a). No major differences are observed for the different temperatures since NEP reaches a minimum values in the $20-200 \mathrm{~Hz}$ range, with values of $2.3 \times 10^{-11} \mathrm{~W} \cdot \mathrm{Hz}^{-}$ $1 / 2$ at $300 \mathrm{~K}$ and $1.7 \times 10^{-11} \mathrm{~W} \cdot \mathrm{Hz}^{-1 / 2}$ at $240 \mathrm{~K}$. Even if the responsivity is much lower at room temperature than at $240 \mathrm{~K}$, the NEP value is of the same order because of the lower bias current necessary to reach the maximum of $\frac{\mathrm{dR}}{\mathrm{dT}}(\mathrm{T}, \mathrm{I})$, which results in lower noise at low frequency. Our NEP values are state-of-the-art for uncooled detectors as reported in Table I and [36, 37] . It should be noted that they rely on the intrinsic properties of the LSMO bolometer, i.e., without any absorbing layers or antennas, and that they are obtained at a quite reduced power bias consumption $(\sim 45 \mu \mathrm{W})$, which could be useful for internet of things (IoT) technologies.

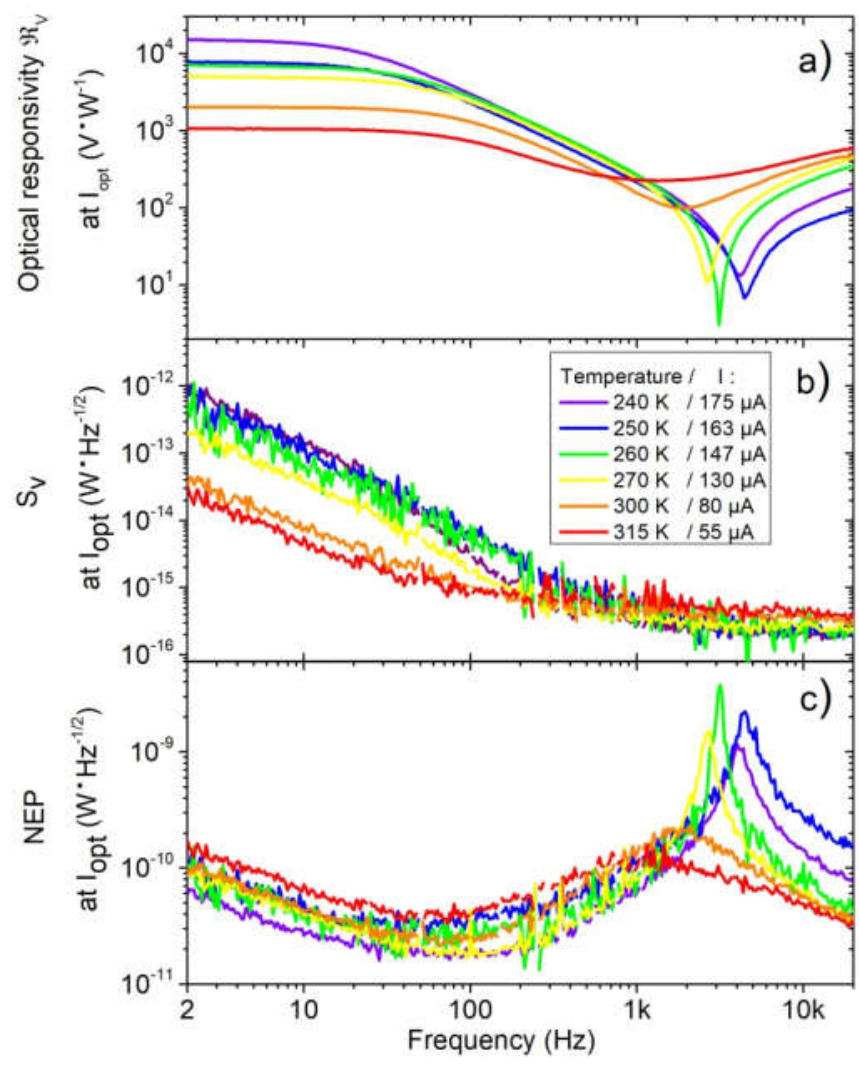

Figure 6: (a) Optical responsivity $\mathfrak{R}_{\mathrm{V}}$; (b) noise power spectral density in voltage; (c) NEP measured versus frequency at different temperatures and at optimal bias currents. The legend applies to all three graphs. 


\section{Conclusion}

In summary, we fabricated and studied an LSMO-based bolometer with a $100 \times 100 \mu \mathrm{m}^{2}$ detection area consisting of 17 parallel suspended lines. Measurements of the V-I characteristics over a range of temperature allowed us to calculate the electrical responsivity of this LSMO bolometer and determine the optimal current at which it was maximal. The optical responsivity was measured under illumination of a laser diode at $635 \mathrm{~nm}$ and confirms the validity of the firstorder model used to calculate the electrical responsivity. Responsivity was higher at low temperature $(240 \mathrm{~K})$, but at a higher optimal current, which was detrimental to the deduced NEP values. The NEP is determined to be of the order of $2-4 \times 10^{-11} \mathrm{~W} \cdot \mathrm{Hz}^{-1 / 2}$ with a time constant of $1.3 \mathrm{~ms}$ at $300 \mathrm{~K}$ for modulation frequencies in $20-200 \mathrm{~Hz}$ range and at $80 \mu \mathrm{A}$, which is very competitive compared to existing uncooled technologies. We anticipate that the performance of these LSMO suspended structures can be further improved by optimizing the radiation-todetector coupling at the desired wavelength using absorbing layers or antennas.

\section{Acknowledgments}

We gratefully acknowledge support from Samsung Electronics Company for the MBE synthesis work performed at Cornell.

\section{Author's contributions}

V.M. and S.L. fabricated the device and measured the electrical, optical and noise characteristics. C.A. grew the LSMO/CTO/Si epitaxial heterostructure with advice from D.G.S. A.A. contributed to the experimental set-up. All authors discussed the results and contributed to the writing of this manuscript.

\section{References}

[1] A. Karim and J.Y. Andersson, IOP Conf. Series: Materials Science and Engineering 51 012001 (2013).

[2] S.S. Dhillon, M.S. Vitiello, E.H. Linfeld et al., J. Phys. D: Appl. Phys. 50043001 (2017).

[3] C.L. Tan, H. Mohseni, Nanophotonics, 7(1): 169-197 (2018). 
[4] R A Lewis, A review of terahertz detectors, J. Phys. D: Appl. Phys. 52433001 (2019).

[5] A. Rogalski, M. Kopytko, and P. Martyniuk, Appl. Phys. Rev. 6, 021316 (2019).

[6] P. L. Richards, J. Appl. Phys. 76, (1994).

[7] P.W. Kruse, L.D. McGlauchlin, R.B. McQuistan, Elements of Infrared Technology, Wiley, New York, NY, (1962).

[8] http://www.tydexoptics.com/pdf/Golay_Detectors.pdf.

[9] W. Li, J. Wang, J. Gou, Z. Huang and Y.J. Jiang, Infrared Millim. THz Waves, 36 42-82 (2015).

[10] https://www.toptica.com/products/terahertz-systems/accessories/schottky-receivers/.

[11] H.W. Hou, Z. Liu, J.H. Teng, T. Palacios and S.J. Chua, Sci. Rep. 746664 (2017).

[12] N. Hiromoto, A. Tiwari, M. Aoki, H. Satoh, M. Takeda and H. Inokawa, 39th International Conference on Infrared, Millimeter, and Terahertz waves (IRMMW-THz), Tucson, AZ, 2014, pp. 1-2 (2014).

[13] Q. Cheng, S. Paradis, T. Bui, and M. Almasri, IEEE Sens. J. 11 (1) 167 (2011).

[14] X.-M. Liu, H.-J. Fang, L.-T. Liu, Microelectronics J. 38735 (2007).

[15] A. Kosarev, S. Rumyantsev, M. Moreno, A. Torres, S. Boubanga and W. Knap, Solid-State Electron. 54 417-9 (2010).

[16] A. Urushibara, Y. Moritomo, T. Arima, A. Asamitsu, G. Kido, and Y. Tokura. Phys. Rev. B 51,14103 (1995).

[17] C. Chen, X. Yi, J. Zhang, B. Xiong, Int. J. Infrared Milli. Waves, 22, 53-58 (2001).

[18] S. Bevilacqua, S. Cherednichenko, IEEE Trans Terahertz Sci Technol, 4 (6) 653 (2014).

[19] V.Y. Zerov, V.G. Malyarov, J. Opt. Technol. 68, 939 (2001).

[20] C. Adamo, L. Méchin, T. Heeg, M. Katz, S. Mercone, B. Guillet, S. Wu, J.-M. Routoure, J. Schubert, W. Zander, R. Misra, P. Schiffer, X. Q. Pan, and D. G. Schlom, APL Materials 3 062504 (2015).

[21] J. W. Reiner, A. M. Kolpak, Y. Segal, K. F. Garity, S. Ismail-Beigi, H. Ahn, and F. J. Walker, Adv. Mater. 22, 2919 (2010).

[22] L. Méchin, C. Adamo, S. Wu, B. Guillet, S. Lebargy, C. Fur, J.-M. Routoure, S. Mercone, M. Belmeguenai, and D. G. Schlom, Physica Status Solidi A Vol. 209 Issue 6 1090-1095 (2012). 
[23] J. M. Vila-Fungueiriño, R. Bachelet, G. S-Girons, M. Gendry, M. Gich, J.Gazquez, E. Ferain, F. Rivadulla, J.R. Carvajal, N. Mestres and A. C. Genevrier, Front. Phys, 338 1-12 (2015).

[24] S. Liu, B. Guillet, A. Aryan, C. Adamo, C. Fur, J.-M. Routoure, F. Lemarié, D.G. Schlom, L. Méchin, Microelectron. Eng. 111, 101-104 (2013).

[25] S. Liu, B. Guillet, C. Adamo, V. M. Nascimento, S. Lebargy, G. Brasse, F. Lemarie, J. El Fallah, D.G. Schlom, and L. Méchin, J. Micromech. Microeng. 29065008 (2019).

[26] A. Aryan, B. Guillet, J.M. Routoure, C. Fur, P. Langlois, L. Méchin, Appl. Surf. Sci., 326, 204-210 (2015).

[27] J. M. Routoure, S. Wu, C. Barone, L. Méchin, B. Guillet, IEEE Transactions on Instrumentation and Measurement, 69 (1) 194 (2020).

[28] P.W. Kruse, L.D. McGlauchlin, R.B. McQuistan, Elements of Infrared Technology, Wiley, New York, NY, (1962).

[29] H. Budzier, G. Gerlach, Thermal Infrared Sensors, Chichester, United Kingdom, (2011).

[30] A. Rogalski, Infrared Detectors 2nd ed, (CRC Press, Boca Raton, USA, 2011).

[31] P. Graziosi, , A. Gambardella, M. Prezioso, A. Riminucci, I. Berggenti, N. Homonnay, G. Schimidt, D. Pullini, D. Busquets-Mataix, Phys. Rev.. B 89, 214411, (2014).

[32] L. Méchin, J.C. Villégier, D. Bloyet, J. Appl. Phys. 81 (10), 7039-7047 (1997)

[33] V. Ceriale, P. Pellegrino, N. Manca, D. Marr, J. Appl. Phys., 115, 054511 (2014).

[34] A. Goyal, M. Rajeswari, R. Shreekala, S.E. Lofland, S.M. Bhagat, T. Boettcher, C. Kwon, T. Venkatesan, Appl. Phys. Lett. 71, 2535-2537 (1997).

[35] L. Méchin, J.M. Routoure, B. Guillet, F. Yang, S. Flament, D. Robbes, R.A. Chakalov, Appl. Phys. Lett. 87204103 (2005).

[36] A. Voshell, N. Dhar, M.M. Rana, Proc. of SPIE Vol. 10209 102090M-1 (2017)

[37] M. Kimata, IEEJ Trans 13: 4-12 (2018). 